\title{
Bacitracin resistance and enhanced virulence of Streptococcus suis via a novel efflux pump
}

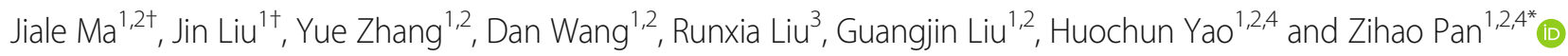

\begin{abstract}
Background: Streptococcus suis is a prominent pathogen causing septicemia and meningitis in swine and humans. Bacitracin is used widely as a growth promoter in animal feed and to control the spread of necrotic enteritis in most developing countries. This study aimed to characterize a novel membrane transporter module Sst comprising SstE, SstF, and SstG for bacitracin resistance.

Results: Comparative genomics and protein homology analysis found a potential efflux pump SstFEG encoded upstream of well-known bacitracin-resistance genes bceAB and bceRS. A four-fold decrease in bacitracin susceptibility was observed in sstFEG deletion mutant comparing with S. suis wildtype strain CZ130302. Further studies indicated that the bacitracin tolerance mediated by SstFEG is not only independent of the BceAB transporter, but also regulated by the two-component system BceSR. Given that SstFEG are harbored by almost all virulent strains, but not in the avirulent strains, we managed to explore its potential role in bacterial pathogencity. Indeed, our results showed that SstFEG is involved in S. suis colonization and virulence in animal infection model by its potential competitive survival advantage against host bactericidal effect.
\end{abstract}

Conclusion: To our knowledge, this is the first study to functionally characterize the bacitracin efflux pump in $S$. suis to provide evidence regarding the important roles of the novel ABC transporter system SstFEG with respect to drug resistance and virulence.

Keywords: Bacitracin, Virulence, Serotype Chz, Streptococcus suis, SstFEG, Efflux pump

\section{Background}

Streptococcus suis is an emerging zoonotic pathogen imposing a serious burden on the porcine industry as well as a severe public health concern $[1,2]$. It is found extensively in porcine breeding environments, which are considered as a potential natural reservoir of resistance genes among several bacteria [3-5]. Bacitracin produced by Bacillus licheniformis is a type of narrow-spectrum peptide acting on Gram-positive bacteria [6]. Bacitracin was previously used as a growth-promoting supplement in animal feed [7]. Furthermore, bacitracin can effectively control necrotic

\footnotetext{
*Correspondence: panzihao@njau.edu.cn

†Jiale Ma and Jin Liu are co-first authors of the article.

${ }^{1}$ College of Veterinary Medicine, Nanjing Agricultural University, Nanjing 210095, China

${ }^{2}$ OIE Reference Laboratory for Swine Streptococcosis, Nanjing 210095, China Full list of author information is available at the end of the article
}

enteritis [8], which is used as a preparation in numerous countries. Prolonged use of bacitracin in animals increases resistance genes in microorganisms. Some molecular mechanisms underlying bacitracin resistance in bacteria have been reported [9-11].

In one such pathway, the Bce system comprising a two-component system (TCS) BceSR and the membrane transporter BceAB to export bacitracin [12]. Similar pathways have been identified in Bacillus subtilis [6], Streptococcus mutants [13], Enterococcus faecalis [9], and Clostridium perfringens [14]. Furthermore, Escherichia coli and Staphylococcus aureus highly express undecaprenol kinase to convert undecaprenol pyrophosphate to undecaprenol phosphate. Some organisms produce exopolysaccharides, such as Streptococcus mutans, or membrane-bound cell-surface phospholipids, such as Xanthomonas campestris [15], which could bind bacitracin to remain extracellular. Bce systems are widely

(c) The Author(s). 2019 Open Access This article is distributed under the terms of the Creative Commons Attribution 4.0 International License (http://creativecommons.org/licenses/by/4.0/), which permits unrestricted use, distribution, and 
distributed in bacteria as a bacitracin efflux pump. The Bce system functions as an importer, as revealed through structural studies. In Bce systems, the RS regulon functions as the key bacitracin-resistance locus; the other module $A B$ pumps out bacitracin via a transmembrane channel. The present study aimed to characterize a novel membrane $\mathrm{ABC}$ transporter module Sst comprising three components SstF, SstE, and SstG, and their role in the regulation of bacitracin resistance. We generated a series of mutant strains to compare their differences in bacitracin resistance with the wild-type strain through a drug sensitivity test and qRT-PCR analysis for bceRS, $b c e A B$, and sstFEG genes. Mice challenge experiments testing bacterial colonization and survival rate were further performed to assess the pathogenicity of the Sst FEG modules in strain CZ130302.

\section{Results}

\section{A potential efflux pump for drug-resistance encoded by} sstFEG

Recently, we reported a highly virulent $S$. suis strain CZ130302 designated as the new serotype Chz causing acute meningitis in piglets. Unexpectedly, CZ130302 also displayed a more extensive drug resistance pattern than S. suis serotype 2 (SS2) reference strains $\mathrm{P} 1 / 7$ and HA9801 in the MIC test, including bacitracin (Table 1). Whole genome retrieval of CZ130302 (CP024974.1) identified a gene cluster encoding the well-known bacitracin transporter BceAB and the related TCS BceSR (Fig. 1a). Further genetic annotation and prediction revealed a novel $A B C$ transporter located at the upstream of $b c e A B$, which was predicted as a potential efflux pump for drug-resistance. Thus, three genes, CVO91_ 06470, CVO91_06465, and CVO91_06460, were designated as $s s t F E G$ for further studies.

\section{SstFEG mediates bacitracin tolerance in S. suis}

Subsequently, a series of deletion mutants were constructed via homologous replacement with a spectinomycin (Spc)

Table 1 The MICs of CZ130302 to different kinds of antibiotics

\begin{tabular}{llll}
\hline Antibiotics & $\mathrm{MIC}(\mathrm{ng} / \mu \mathrm{L})^{\mathrm{a}}$ & Antibiotics & $\mathrm{MIC}(\mathrm{ng} / \mu \mathrm{L})^{a}$ \\
\hline Penicillin G & 16 & Tetracycline & 128 \\
Ampicillin & 64 & Doxycycline & 32 \\
Streptomycin & 256 & Erythromycin & 512 \\
Gentamicin & 256 & Lincomycin & 512 \\
Kanamycin & $>512$ & Bacitracin & 64 \\
Spectinomycin & 32 & Vancomycin & 0.25 \\
Amikacin & 128 & Nisin & 256 \\
Neomycin & $>512$ & Ciprofloxacin & 64 \\
Chloramphenicol & 4 & Norfloxacin & 128 \\
\hline
\end{tabular}

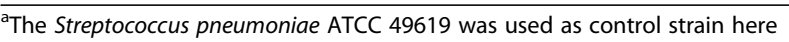

resistance expression cassette [16]. Construction of the mutant strains was confirmed via PCR (Additional file 1). The bacterial growth of CZ13- $\triangle$ SstFEG showed $1 \mathrm{~h}$ delay in logphase compared with that of wild-type strain (Additional file 2), but no significant difference in the last stage. It should be noted that the bacitracin sensitivity of different mutant strains was increased at varying degrees in comparison with the wild-type strain (Fig. 2a). The mutant strain CZ13- $\triangle$ sstFEG (MIC, $16 \mu \mathrm{g} / \mathrm{mL}$ ) was approximately 4-fold more sensitive to bacitracin than the wild-type CZ130302 strain was (MIC, $64 \mu \mathrm{g} / \mathrm{mL}$ ), concurrent with the colony growth of CZ13- $\triangle$ sstFEG on the culture media supplemented with bacitracin at different concentrations (Fig. 2b). Otherwise, CZ13- $\triangle b c e A B$ and CZ13- $\triangle b c e R S$ (MIC, $4 \mu \mathrm{g} /$ $\mathrm{mL})$ were more sensitive to bacitracin than CZ13- $\triangle$ sstFEG was (MIC, $16 \mu \mathrm{g} / \mathrm{mL}$ ). Based on the MIC, bacterial growth inhibition at $8 \mathrm{~h}$ was compared upon bacitracin supplementation at high concentrations in the log-phase. Obviously, the mutant strain CZ13- $\triangle b c e A B$ displayed the greatest reduction in bacterial survival at each time point (Fig. 2c). In summary, both BceAB and SstFEG transporters co-regulate bacitracin resistance in strain CZ130302, but their functional correlation in this process is unclear.

\section{Bacitracin tolerance mediated by SstFEG is independent of the BceAB transporter}

To further understand the functional characteristics of SstFEG, BceAB, and BceRS when bacterial cells responded to bacitracin stress, total RNAs of wild-type and mutant strains were extracted under the same culture conditions with or without bacitracin treatment, and the expression of relevant seven genes (sstFEG/bceAB/bceRS) were comparatively analyzed via qRT-PCR. As shown in Fig. 3a, the bacitracin supplementation in culture medium significantly activated the expression of sstFEG and bceAB at least 30fold higher than that in the normal culture. However, the transcriptional level of $b c e R S$ was not significantly upregulated under this condition. These results indicated that the bceRS and sstFEG/bceAB were controlled by different operons, suggesting that the following study need to be performed under the bacitracin treatment to confirm the function of SstFEG in bacitracin resistance directly or indirectly.

Indeed, our previous genetic analysis showed that sstFEG, bce $A B$, and bceRS are controlled by different operons (Fig. 1a), respectively. Here, we need to confirm whether the deletion of sstFEG caused the expression change of bceAB and $b c e R S$, which have been reported for bacitracin resistance in several bacterial species [12]. As shown in Fig. 3b, the deletion of sstFEG did not downregulate the expression of $b c e A B R S$ and even caused the significant activation of $b c e A$ by more than 4 -fold. These results suggested that the deficiency of bacitracin resistance caused by sstFEG deletion does not relate with well-known BceAB and BceSR systems. Unexpectedly, the deletion of bceAB significantly 
A
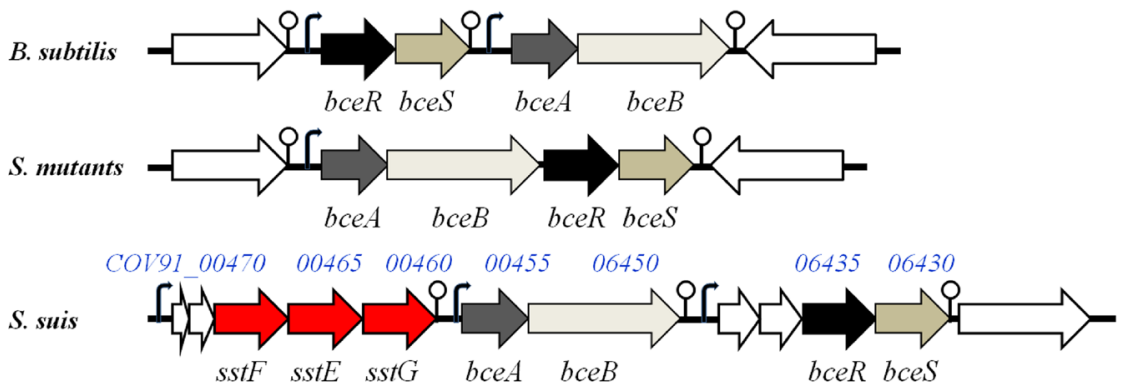

B

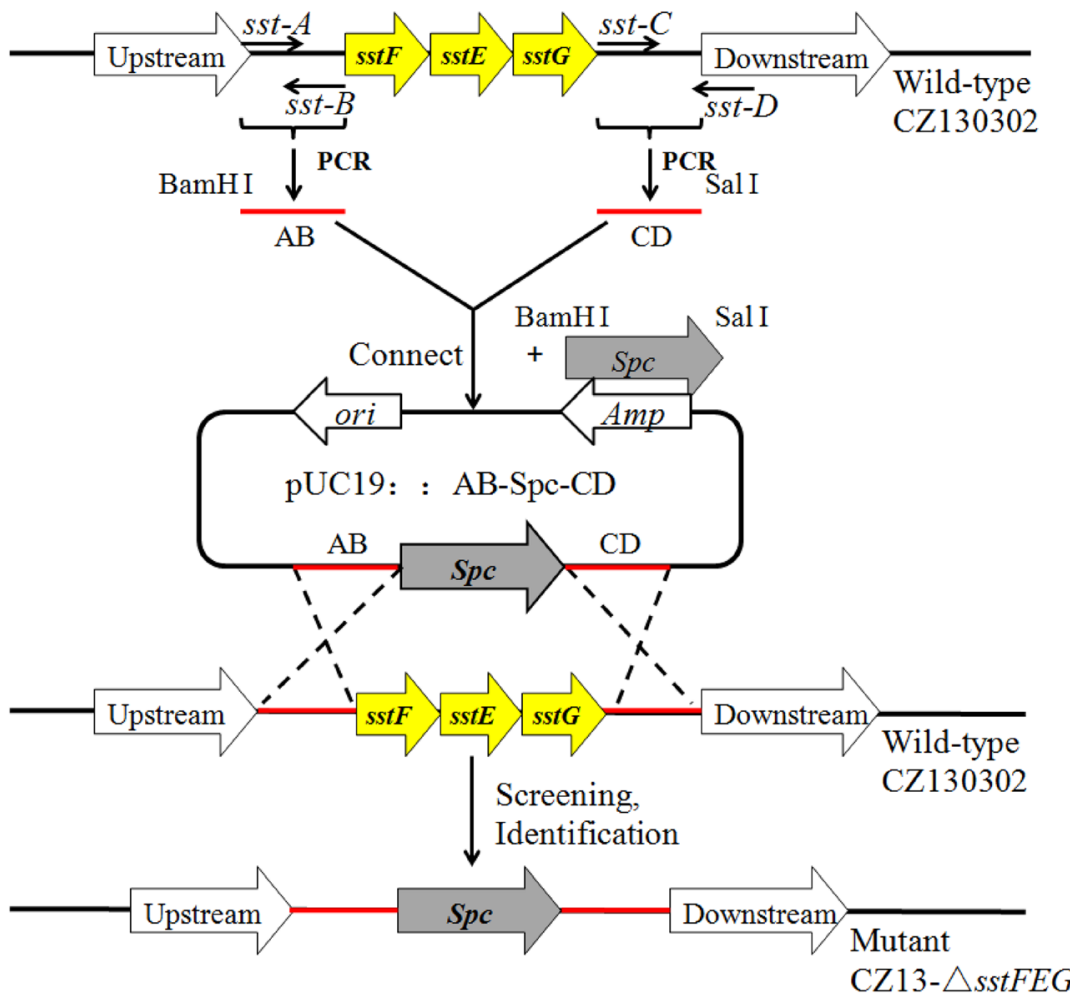

Fig. 1 The genetic neighborhood analysis and the mutant construction strategy. a A schematic representation of the genetic locus containing seven genes (CVO91_06470 to CVO91_06430), along with the adjacent genes, comparing gene organization characteristics in Bacillus subtilis, Streptococcus mutans, and S. suis. The genes bceABSR are ubiquitous, while sstFEG are exclusively present in S. suis. Sequence analysis of this genetic locus indicates three potential transcriptional terminators in the region between sst $G$ and bceA and downstream of bceB and bces. $\mathbf{b} A$ schematic representation of mutant construction strategy. Three fragments $A B, S p c$, and CD were fused to form an intermediate vector with pUC19 plasmid. The mutant strains were obtained via natural genetic transformation, screening, and identification.

inhibited the expression of sstFEG (Fig. 3c), suggesting that the deficiency of bacitracin resistance caused by bceAB deletion may be related with BceAB inactivation and sstFEG downregulation together. These observations, together with the results shown in Fig. 2, further implied that SstFEG is an efflux pump for bacitracin and can transport bacitracin independently.

\section{BceSR regulates SstFEG in response to bacitracin resistance}

Here, we explored why the deletion of $b c e A B$ inhibited the expression of sstFEG (Fig. 3c), and found that bceRS is downregulated by approximately $50 \%$ in the $\triangle b c e A B$ mutant. As revealed from the KEGG Pathway Database (Additional file 3) and previous studies [11], BceAB transporter serves as an efflux pump along with the BceSR TCS to regulate bacitracin perception. Thus, it is reasonable to speculate that BceSR also is the important regulator of sstFEG operon. Indeed, the deletion of $b c e R S$ caused significant downregulation of sstFEG and $b c e A B$ more than 50-fold (Fig. 3d), which almost lost the transcriptional activation of these genes in response to bacitracin stress under the $2 \mathrm{ng} / \mu \mathrm{L}$ concentration, indicating that BceSR is the major regulator for bacitracin 
A

\begin{tabular}{|c|c|c|c|c|}
\hline \multirow[b]{2}{*}{ Antibiotic } & \multicolumn{4}{|c|}{$\operatorname{MIC}(\mu \mathrm{g} / \mathrm{mL})$} \\
\hline & CZ130302 & CZ13- $\Delta s s t E F G$ & CZ13- $\triangle b c e A B$ & CZ13- $\Delta b c e R S$ \\
\hline Bacitracin & 64 & 16 & 4 & 4 \\
\hline
\end{tabular}

B
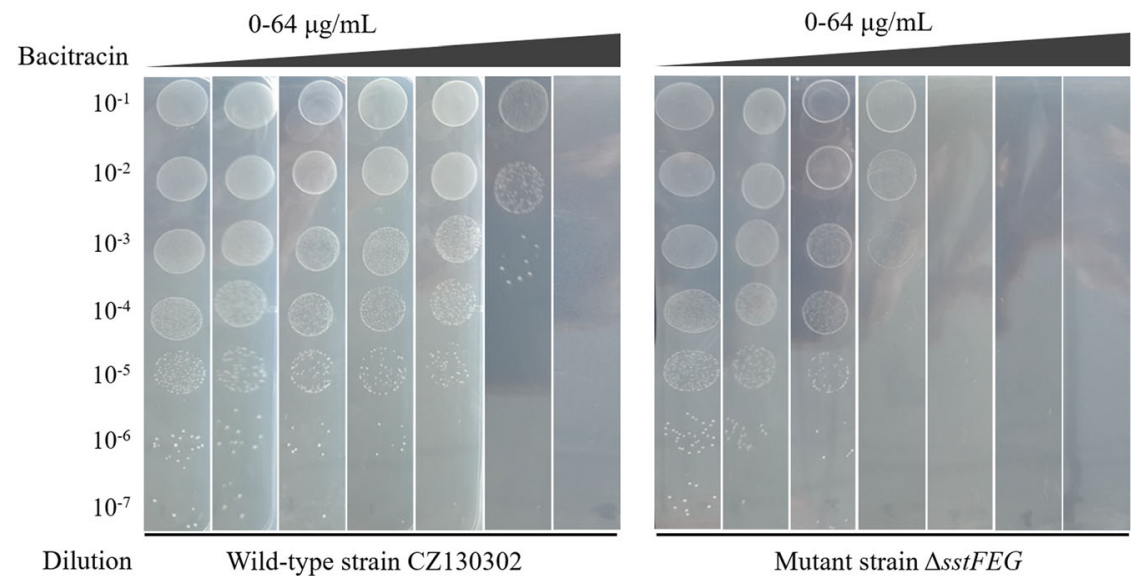

C

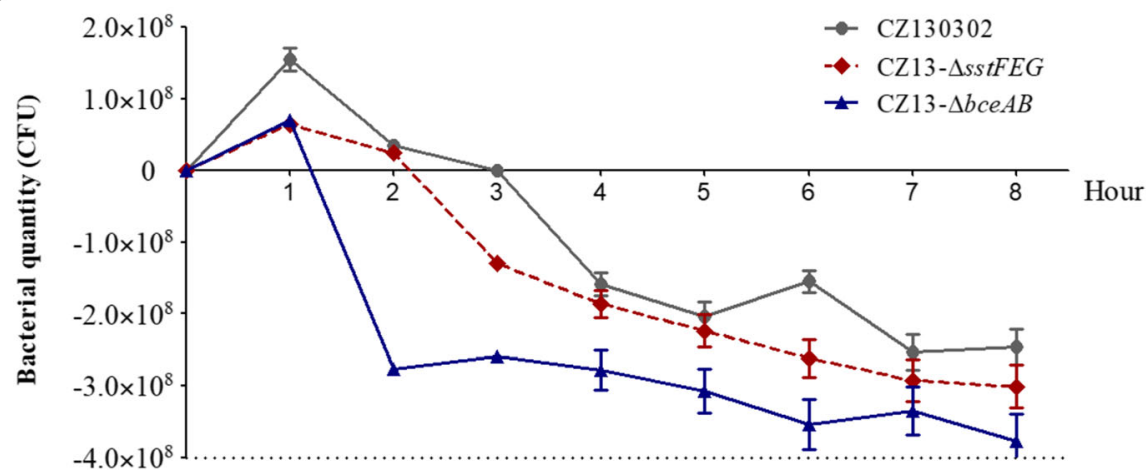

Fig. 2 Bacitracin sensitivity of the mutant strains. a Comparison of MICs to bacitracin between wild-type and mutant strains. $\mathbf{b}$ The bacterial colony growth in THB plate with bacitracin resistance. Bacterial cells in the logarithmic phase were subjected to serial dilution and $10 \mu \mathrm{L}$ of bacterial cultures were in THB plates supplemented with bacitracin at different concentrations $(0,2,4,8,16,32$, and $64 \mu \mathrm{g} / \mathrm{mL})$. c The bacterial survival of wild-type and mutant strains incubated with high bacitracin concentration. The bacterial cells were eliminated with $128 \mu \mathrm{g} / \mathrm{mL}$ bacitracin. We monitored and recorded the bacterial loads incubated after $8 \mathrm{~h}$ with $128 \mu \mathrm{g} / \mathrm{mL}$ bacitracin in the logarithmic phase. The CFU values of all mutants was reduced to a greater extent in comparison with the wild-type strain at each hour

resistance in this case. These results suggested that the downregulation of $b c e R S$ caused by the deletion of $b c e A B$ may be the primary reason of $s s t F E G$ transcriptional inhibition, and sstFEG regulation by BceSR.

\section{S. suis colonization and virulence requires bacitracin tolerance}

The prevalence of sstFEG, bceAB, and bceSR genes in 35 whole genomes of $S$. suis from NCBI database were investigated, which showed that all strains encode $b c e A B$ and $b c e R S$ genes, but only 19 strains (18 highly virulent or virulent strains except for T15) encode sstFEG genes, and almost all avirulent strains are sstFEG negative
(Table 2). In particular, serotype 3 strain ST3, isolated from a pig with pneumonia in Hubei province in 2009 [43], only harbors $s s t F$, but not $s s t E$ and $s s t G$. These results suggested that SstFEG may be involved in the pathogenicity of S. suis.

The colonization of S. suis requires to antagonize mucosal microflora and host immune clearance. Many bacterial species from mucosal microflora can secrete bacitracin to achieve competitive advantage for optimal survival. Furthermore, bacitracin was previously used as a growthpromoting supplement in animal feed. Here, we managed to test whether SstFEG efflux pump is required for S. suis original colonization. Indeed, the inactivation of SstFEG 


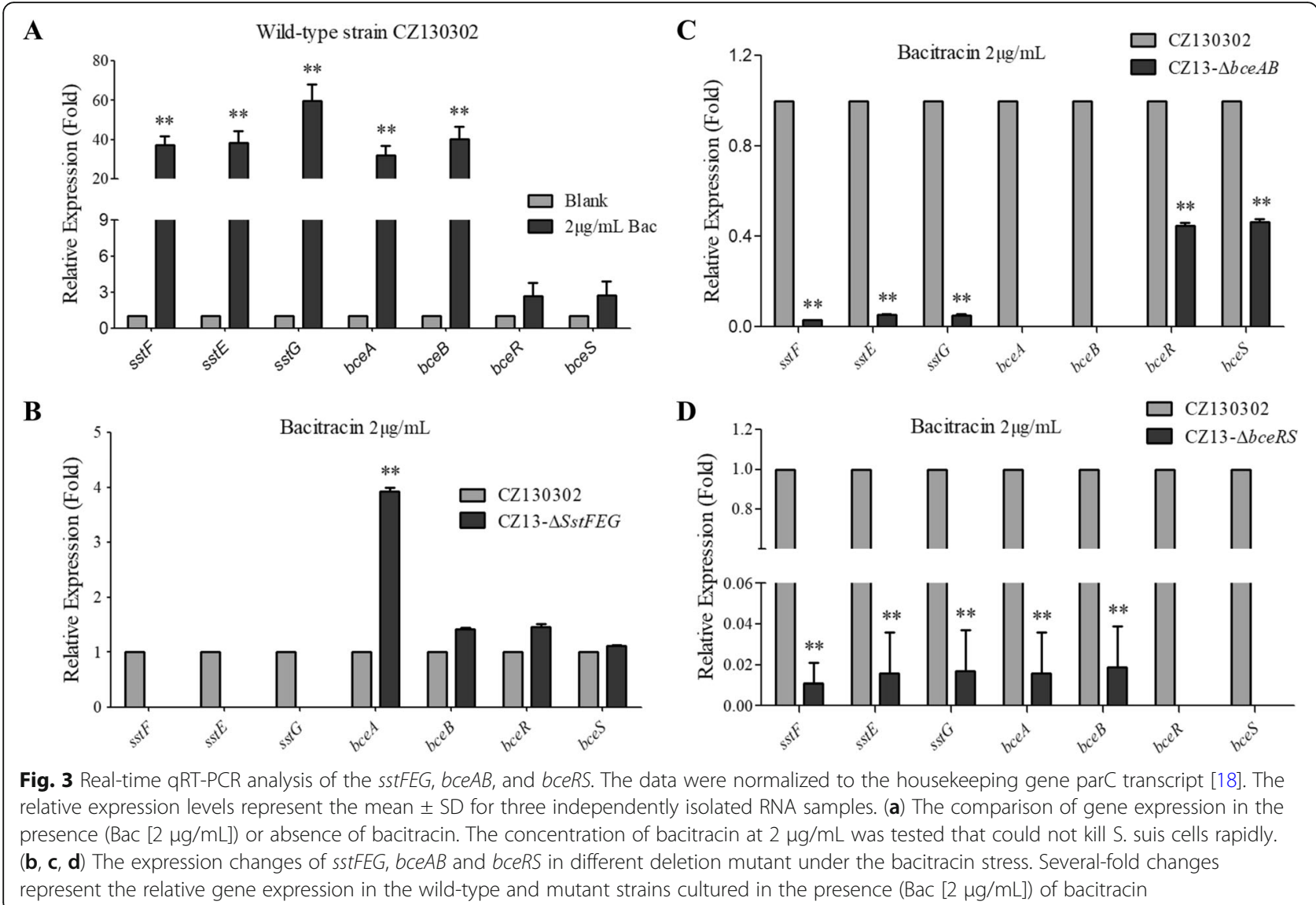

resulted in a 2- to 3 -fold reduction in the bacterial loads compared to the wild-type strain in blood after intraperitoneal injection for $10 \mathrm{~h}$ (Fig. 4a), indicating that SstFEG mediating bacitracin tolerance are essential for $S$. suis to rapidly infect the host. As shown in Fig. 4b, mice infected with the wild-type strain CZ130302 had severe clinical symptoms and displayed $100 \%$ mortality on the fifth day after challenge. In contrast, the group infected with the mutant CZ13- $\triangle$ sstFEG strain displayed a $50 \%$ greater survival rate $(5 / 11)$.

\section{Discussion}

Thus far, the underlying mechanism of bacitracin resistance has been reported in B. subtilis, S. mutans, and $S$. pneumoniae, but not in $S$. suis. The present study shows that the network of bacitracin resistance in Streptococcus partially comprises TCS and ABC transporters, as revealed from the KEGG Pathway Database (Additional file 3). BceSR (TCS) and BceAB (ABC transporter) are ubiquitous and significantly influence the bacitracin resistance of Streptococcus spp. In this study, we identified a novel ABC transport module (CVO91 06470, 06465, and 06460) mediating bacitracin resistance and virulence in $S$. suis. The CVO91 06470 gene encodes an ABC transporter ATPbinding protein that shares approximately $42 \%$ protein homology with the BcrA system, contributing to bacitracin resistance in B. subtilis.

Interestingly, these three genes (designated as sstFEG) are coincidentally located upstream of the four genes encoding well-known BceAB and BceSR homologs (Fig. 1a). Bacitracin sensing depends on the BceRS signal transduction system and the BceAB transporter as a co-sensor. Jing Ouyang et al [12] reported that phosphorylated BceR upregulates the positively regulated expression of the bceABRS operon. In this study, we proposed that BceAB, BceRS, and SstFEG may constitute a seven-component system to regulate the bacitracin efflux optimally. Our results indicated that the bacitracin tolerance mediated by SstFEG is not only independent of the BceAB transporter, but also regulated by the TCS BceSR. Furthermore, sstFEG and $b c e A B$ are controlled by different operons, but the deletion of $b c e A B$ caused significant downregulation of bceRS, which decreased the bacitracin resistance mediated by SstFEG by inhibiting the transcriptional activation. In contrast, the deletion of sstFEG could not regulate the BceAB and BceSR systems for sensing bacitracin. A model for this regulatory pathway of bacitracin resistance in S. suis is proposed (Fig. 5). 
Table 2 The distribution of seven bacitracin resistance genes in Streptococcus suis

\begin{tabular}{|c|c|c|c|c|c|c|c|c|}
\hline Strains & Serotype $^{a}$ & Virulence & host & Symptoms & $B c e B / A$ & Bces/R & sstFEG & Reference \\
\hline CZ130302 & Chz & Highly virulent & piglet & Meningitis & + & + & + & [18] \\
\hline BM407 & 2 & Highly virulent & human & STSS & + & + & + & [19] \\
\hline 05ZYH33 & 2 & Highly virulent & human & STSS & + & + & + & [20] \\
\hline $98 \mathrm{HAH} 33$ & 2 & Highly virulent & human & STSS & + & + & + & [20] \\
\hline GZ1 & 2 & Highly virulent & human & STSS & + & + & + & [21] \\
\hline SC84 & 2 & Highly virulent & human & STSS & + & + & + & [19] \\
\hline SC19 & 2 & Highly virulent & piglet & Meningitis & + & + & + & [22] \\
\hline 05ZY719 & 2 & Highly virulent & piglet & Septicemia & + & + & + & [23] \\
\hline SC070731 & 2 & Highly virulent & piglet & Meningitis & + & + & + & [24] \\
\hline P1/7 & 2 & Highly virulent & pig & Septicemia & + & + & + & [25] \\
\hline S735 & 2 & Highly virulent & pig & Septicemia & + & + & + & {$[26]$} \\
\hline A7 & 2 & Highly virulent & pig & Septicemia & + & + & + & [27] \\
\hline CS100322 & 2 & Virulent & pig & lung & + & + & + & {$[28]$} \\
\hline SS2-1 & 2 & Virulent & pig & diseased & + & + & + & [29] \\
\hline Т15 & 2 & Avirulent & pig & Septicemia & + & + & + & {$[30]$} \\
\hline SS12 & $1 / 2$ & Virulent & pig & lung & + & + & + & [27] \\
\hline JS14 & 14 & Highly virulent & pig & lung & + & + & + & [31] \\
\hline GZ0565 & 9 & Highly virulent & pig & Septicemia & + & + & + & [31] \\
\hline LSM102 & - & Highly virulent & pig & Septicemia & + & + & + & {$[32]$} \\
\hline ST3 & 3 & Virulent & pig & Septicemia & + & + & $+/-$ & [21] \\
\hline AH681 & Chz & Avirulent & pig & Healthy & + & + & - & [18] \\
\hline HN136 & Chz & Avirulent & pig & Healthy & + & + & - & [18] \\
\hline ST1 & 1 & Avirulent & pig & Healthy & + & + & - & [27] \\
\hline HA0609 & 2 & Avirulent & pig & Healthy & + & + & - & [28] \\
\hline NSUI002 & 2 & Avirulent & pig & Healthy & + & + & - & [33] \\
\hline 05HAS68 & 2 & Avirulent & pig & Healthy & + & + & - & [34] \\
\hline NSUI060 & 2 & Avirulent & pig & Healthy & + & + & - & [35] \\
\hline YB51 & 3 & Avirulent & pig & Healthy & + & + & - & {$[36]$} \\
\hline 6407 & 4 & Avirulent & pig & Healthy & + & + & - & [37] \\
\hline D9 & 7 & Avirulent & pig & Healthy & + & + & - & [27] \\
\hline D12 & 9 & Avirulent & pig & Healthy & + & + & - & [38] \\
\hline TL13 & 16 & Avirulent & pig & Healthy & + & + & - & [39] \\
\hline LS9N & - & Avirulent & + & Healthy & + & + & - & [40] \\
\hline 90-1330 & - & Avirulent & + & Healthy & + & + & - & [41] \\
\hline DN13 & 9 & Avirulent & pig & Healthy & + & + & - & [42] \\
\hline
\end{tabular}

Except for the bacitracin resistance, antibiotic susceptibility testing indicated that strain CZ130302 exhibits a more extensive drug resistance pattern than that of other S. suis strains. Comparative genomics studies have reported that the genomes of newly isolated CZ130302 harbor at least $45 \mathrm{ISs}$, including the novel 82-kb integrative conjugative element ICESsuCZ130302 [17], which may increase the potential horizontal gene transfer from different bacterial species or genera. Further studies need to explore how and why strain CZ130302 acquired numerous exogenous antibiotic resistance genes and integrated into a chromosome, especially for newly identified SstFEG efflux pump.

Given that the SstFEG never been reported in any other opportunistic or commensal bacteria species, we speculated that its encoding genes may be transferred from pathogenic bacterial strains. To further examine the correlation between the virulence of $S$. suis strains and SstFEG, genomic data of 34 S. suis strains from GenBank were investigated. Consequently, SstFEG are harbored by 

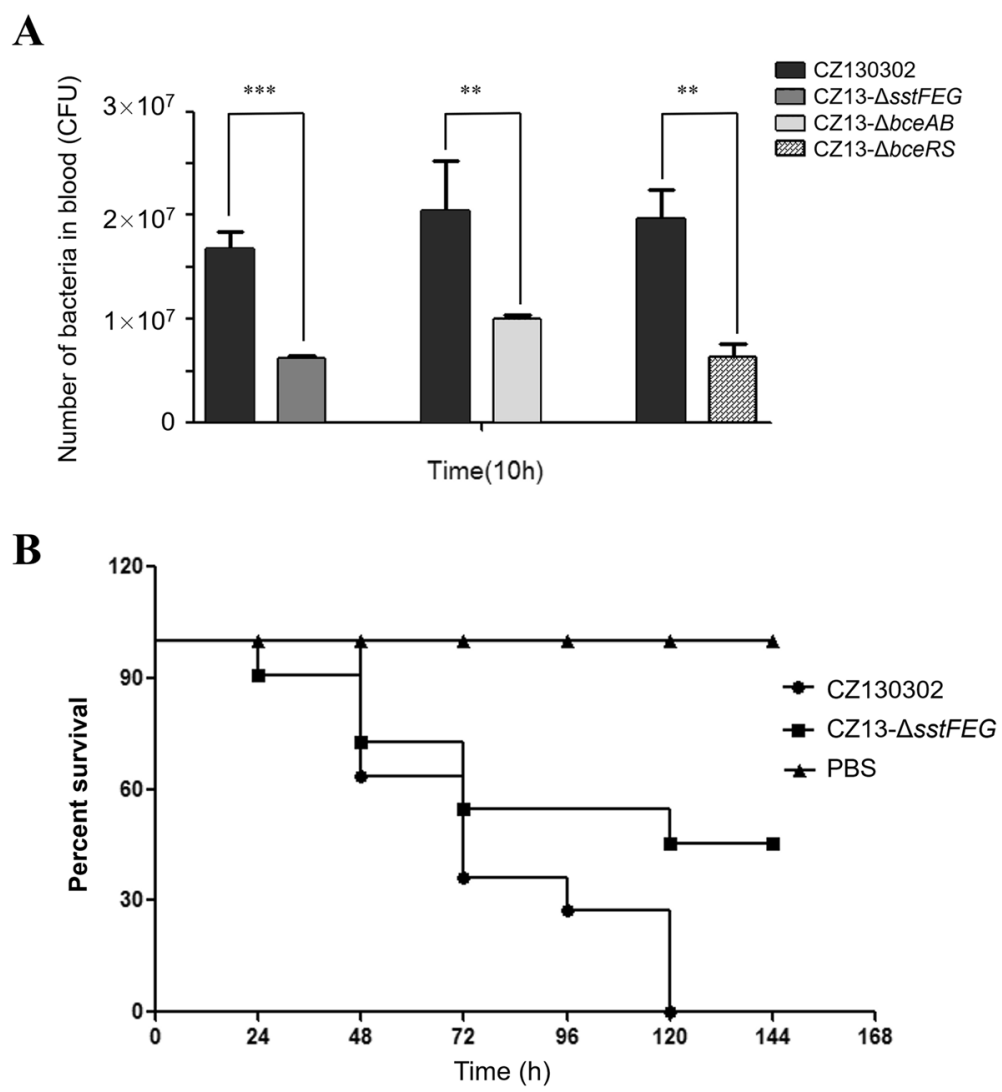

Fig. 4 Bacterial competitive colonization and virulence analysis in vivo. a The competitive test was carried out to compare the colonization between wild-type and mutant strains in mouse infection model. The bacterial loads of mutant strains were decreased by approximately two- or three-fold in comparison with the wild-type strain in blood after intraperitoneal challenge for $10 \mathrm{~h},{ }^{* *} P<0.01$ and ${ }^{* * *} P<0.001$. b Survival curves of BALB/c mice infected with wild-type or mutant strains. Six-week-old female BALB/c mice were segregated into three groups and inoculated i.p. with $2.6 \times 10^{7}$ cells/mouse. Mice infected with the vehicle solution (PBS) were used as controls and survival was monitored over a 7-d period. Data are expressed as the mean percentage of live animals in each group $(n=11)$, $P<0.05$

almost all virulent strains isolated from the patients and diseased pigs, but not in the avirulent strains isolated from healthy pigs. To further explore the potential roles of SstFEG in $S$. suis virulence, BALB/c mouse infection model was employed for challenge tests [44]. Indeed, the pathogenicity of the mutant strain deleted sstFEG was significantly decreased.

\section{Conclusions}

This study reports a novel membrane transporter module SstFEG, which functions as not only an efflux pump for bacitracin resistance, but also a virulence-related protein involved in S. suis pathogenicity.

\section{Methods}

\section{Bacterial strains, plasmids, and culture conditions}

S. suis strain CZ130302 from the novel serotype Chz was isolated from a diseased piglet in Changzhou, China $[18,45]$. Plasmid pSET-4S with Spc resistance gene was generously provided by Professor Daisuke Takamatsu. Plasmid pUC19 was maintained in the OIE Reference
Laboratory for Swine Streptococcosis. S. suis were cultured in Todd Hewitt Broth (THB, BD) or agar comprising $6 \%(\mathrm{v} / \mathrm{v})$ sheep blood at $37^{\circ} \mathrm{C}$ and $5 \% \mathrm{CO}_{2}$. E. coli strains were cultured in Luria-Bertani (LB, BD) medium at $37^{\circ} \mathrm{C}$ supplemented with $100 \mu \mathrm{g} / \mathrm{mL}$ Spc (Sigma) per requirement for S. suis and $50 \mu \mathrm{g} / \mathrm{mL}$ Spc or $100 \mu \mathrm{g} / \mathrm{mL}$ ampicillin (Amp, Sigma) for E. coli. Different types of antibiotics, especially bacitracin (Bac, Sigma), were used to determine the minimum inhibitory concentration. In total, $100 \mu \mathrm{g} / \mathrm{mL}$ lysozyme (Lzm, Sigma) and $1 \mu \mathrm{g} / \mathrm{mL}$ vancomycin (Van, Sigma) were used for phagocytosis assays.

\section{Antimicrobial susceptibility assays}

In accordance with the standardized methods per the Clinical and Laboratory Standards Institute (CLSI) guidelines (2015), the minimum inhibitory concentrations (MICs) of different types of antibiotics, including beta-lactams, aminoglycosides, tetracyclines, amide alcohols, macrolides, lincosamides, polypeptides, and fluoroquinolone against $S$. suis CZ130302 were determined. In 


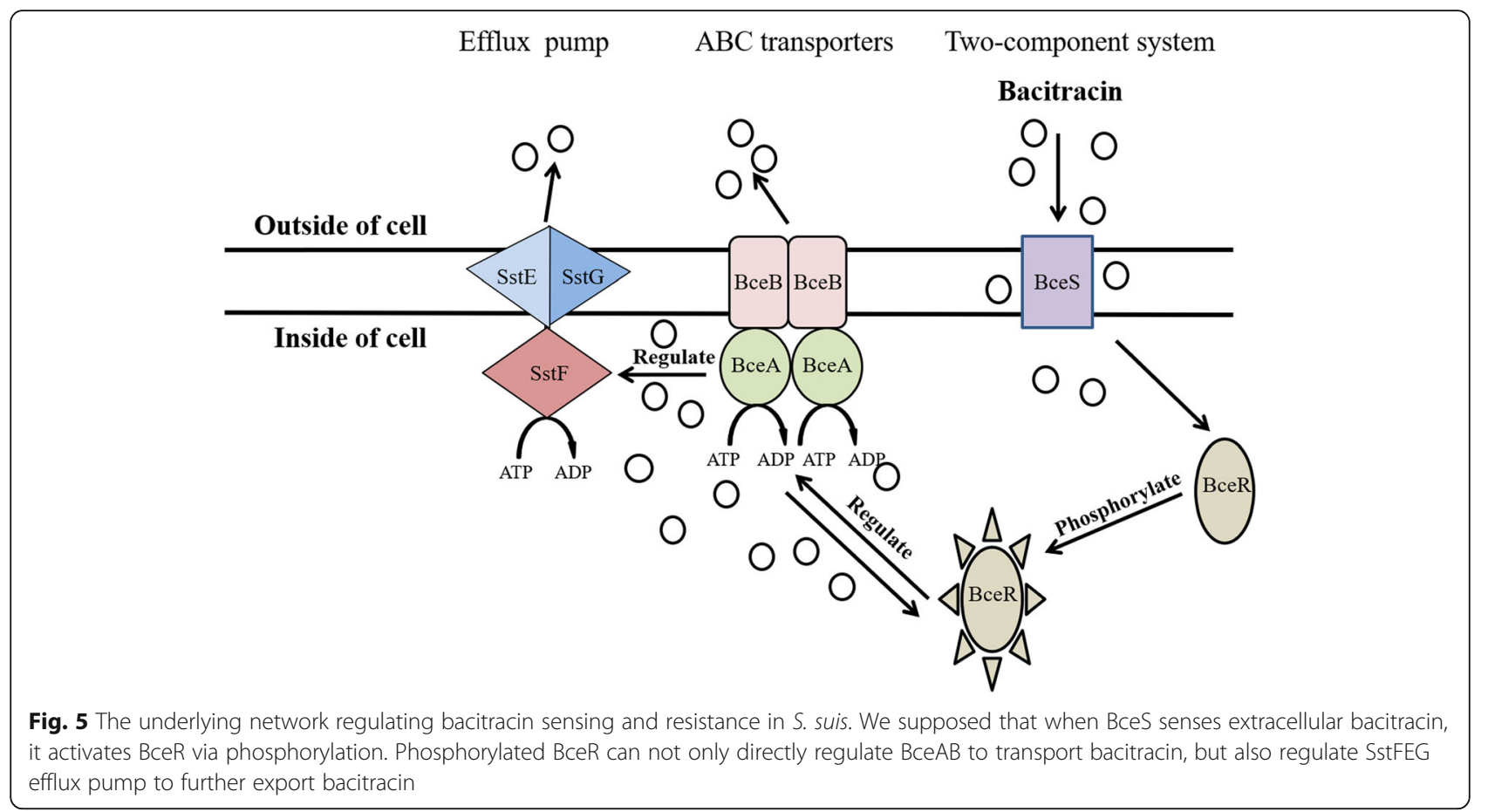

brief, the strains were diluted 1000-fold into Cation Adjusted Muller Hinton Broth (CAMHB) with lysed horse serum $(2.5 \% \mathrm{v} / \mathrm{v})$ and cultured to an optical density at $600 \mathrm{~nm}\left(\mathrm{OD}_{600}\right)$ of approximately 0.5 ; thereafter, $180 \mu \mathrm{L}$ of the culture was inoculated into the first vertical well, while $100 \mu \mathrm{L}$ was inoculated in the other wells. Different initial antibiotic concentrations in $20 \mu \mathrm{L}$ were placed in the first well and mixed, and $100 \mu \mathrm{L}$ of the mixture from the first well was transferred to the subsequent well. This was repeated and as an analogy until the last well. Subsequently, cultures were incubated at $37{ }^{\circ} \mathrm{C}$ and $5 \%$ $\mathrm{CO}_{2}$ for $20 \mathrm{~h}$. CZ130302 was tested through a serial dilution from $10^{-1} \mathrm{CFU}$ to $10^{-7} \mathrm{CFU}$, and the THB plates with a 2-fold dilution of bacitracin, initiating at $64 \mu \mathrm{g} /$ $\mathrm{mL}$. All experiments were performed in triplicate. The Streptococcus pneumoniae strain ATCC 49619 was used as a control to ensure the reliability of tested data.

\section{Prediction and distribution of bacitracin-resistant genes in Streptococcus suis}

The complete genome sequence of $S$. suis strain CZ130302 was obtained from NCBI (https://www.ncbi. nlm.nih.gov/, GenBank: CP024974.1). Antibiotic Resistance Genes Database (ARDB, http://ardb.cbcb.umd. $\mathrm{edu} /$ ) was used to predict resistance genes in the genome of $S$. suis. KEGG PATHWAY Database (http:// www.genome.jp/kegg/pathway.html) was used to extract previously reports pathways underlying bacterial bacitracin resistance and the distribution of resistance genes in $S$. suis exhibited in the NCBI database. In addition, Rockhopper software was used to predict the operon, transcription start site (TSS), and transcription termination sites (TTS) of CZ130302 in accordance with the distribution of reads in the genome.

\section{Construction of gene deletion mutants}

To investigate the contribution of predictable genes, a series of deletion mutants was constructed via natural DNA transformation instead of the traditional method with pSET-4 s because this method was not suitable for S. suis CZ130302. Primers used to construct and confirm the mutants are enlisted in Additional file 4. The construction strategy for sstFEG deletion mutant strain is shown in Fig. 1. In brief, fragment $A B(574 \mathrm{bp})$ and $C D$ (867 bp) were amplified from S. suis CZ130302, using primers sst- $A$ (BamHI site at its $5^{\prime}$ end) and sst- $B$, sst- $C$, and sst- $D$ (Sal I site at its $3^{\prime}$ end) as upstream and downstream of sstFEG, respectively. The $s p c$ sequence (1133 $\mathrm{bp)}$ with the promoter was amplified from plasmid pSET $-4 \mathrm{~s}$ by primers $S p c-F$ and $S p c-R$. These three fragments were fused via PCR and ligated with pUC19 to form the recombinant plasmid pUC19-AB-Spc-CD. The CZ13- $\triangle$ sstFEG mutant was obtained via homologous recombination and resistance screening. Other mutant strains including CZ13- $\triangle$ bce $A B$ and CZ13- $\triangle$ bceRS were constructed as previously described.

\section{Anti-pressure analysis of bacitracin}

sstFEG mutant strains were cultured up to an optical density of approximately 0.7 at $600 \mathrm{~nm}\left(\mathrm{OD}_{600}\right)$. Thereafter, bacitracin was added at a high concentration. All bacteria were cultured for $8 \mathrm{~h}$ at $37^{\circ} \mathrm{C}$ and $5 \% \mathrm{CO}_{2}$ 
continuously, followed by plating of serial ten-fold dilutions on THB agar to enumerate bacteria at each hour. The trial was performed in triplicate, and the results were determined using the following formula: CFUs of viable bacteria at each hour - CFUs of original bacteria.

\section{qRT-PCR analysis}

To analyze the expression levels of related genes and their interactions, total RNA was isolated from CZ130302 and mutant strains upon culturing to an optical density at 600 $\mathrm{nm}\left(\mathrm{OD}_{600}\right)$ of $0.6 \mathrm{in} \mathrm{THB}$ broth, with or without $2 \mu \mathrm{g} / \mathrm{mL}$ bacitracin, using RNAiso Plus (Takara, Japan) in accordance with the manufacturer's instructions. When bacterial strains were cultured with bacitracin, the Streptococcus pneumoniae strain ATCC 49619 was used as control. The resulting RNA was then treated with gDNA Eraser to remove genomic DNA and further converted to cDNA via reverse transcription (RT), using PrimeScript ${ }^{\mathrm{TM}} \mathrm{RT}$ reagent Kit (Takara). cDNA samples were synthesized using RNA species harvested from three independent cultures of each strain. Real-time quantitative PCR assays were performed in triplicate with the method of SYBR Green detection. Primers for qRT-PCR analysis are enlisted in Additional file 4. The relative amount of target gene mRNA was normalized to the housekeeping gene parC [46]. The relative fold change was calculated by the threshold cycle $\left(2^{-\Delta \Delta} C_{T}\right)$ method [47]. Each assay was performed in duplicate in three independent experiments.

\section{Animal experiments}

A competitive test was performed to compare the colonization ability between wild-type and mutant strains in mice. Prior to this experiment, all mice were fed with bacitracin in water to activate bacterial genes. Eighteen female 6-week-old BALB/c mice were equally segregated into three groups. The wild-type and mutant strains were cultured until the $\mathrm{OD}_{600}$ was approximately 0.8 , and the density of each strain was adjusted to $1.3 \times$ $10^{8} \mathrm{CFU}$ in PBS. Thereafter, the wild-type and different mutant strains were respectively mixed in a 1:1 ratio and challenged intraperitoneally with $200 \mu \mathrm{L} /$ mouse. After $10 \mathrm{~h}$, blood was sampled from each mouse, followed by plating serial five-fold dilutions on THB agar, containing $50 \mu \mathrm{g} / \mathrm{mL}$ kanamycin with or without $100 \mu \mathrm{g} / \mathrm{mL}$ Spc to distinguish and enumerate bacteria. In addition, the virulence of mutant CZ13- $\triangle$ Sst FEG strain was assessed in the BALB/c mouse model of infection. Thirty-three female 6-week-old BALB/c mice were equally segregated into three groups and challenged intraperitoneally with $200 \mu \mathrm{L} /$ mouse at approximately $2.6 \times 10^{7} \mathrm{CFU}(10 \times$ $\mathrm{LD}_{50}$ ) in PBS. Mice infected with the vehicle (PBS) were used as controls. Mortality was monitored every $8 \mathrm{~h}$ for 7 d. All experiments were performed in triplicate. All animals used in this study were humanely euthanized by carbon dioxide asphyxiation in an airtight box.

\section{Statistical analyses}

GraphPad Prism version 5 is used to analyze and plot the data. Student's $t$-test (unpaired) was performed to determine differences between the means of the two samples. Differences with a $P$ value of $<0.05$ were considered significant, and a $P$-value of $<0.01$ was considered greatly significant.

\section{Ethics statement}

Six-week-old female germfree BALB/c mice were purchased from the Comparative Medicine Center of Yangzhou University. Animal experiments were carried out in the Laboratory Animal Center of Nanjing Agricultural University and approved by Laboratory Animal Monitoring Committee of Jiangsu Province, China [Permit number: SYXK (SU) 2017-0007].

\section{Supplementary information}

Supplementary information accompanies this paper at https://doi.org/10. 1186/s12917-019-2115-2.

Additional file 1. Identification of mutant strains via PCR.

Additional file 2. Comparison of growth curves between wild-type CZ130302 and mutant strain CZ13- $\triangle$ sstEFG.

Additional file 3. Genes involved in bacitracin transport

Additional file 4. Primers used in this study.

Abbreviations

CLSI: Clinical and Laboratory Standards Institute; LB: Luria-Bertani; MICs: The minimum inhibitory concentrations; SS: Streptococcus suis; TCS: Twocomponent system; THB: Todd Hewitt Broth; TSS: Transcription start site

\section{Acknowledgements}

We would like to thank the participating workers of the OIE Reference Laboratory for Swine Streptococcosis in Nanjing Agricultural University.

\section{Authors' contributions}

ZP conceived and designed the experiments. JM, JL, YZ and DW performed the experiments. GL contributed materials and analysis tools. JM, HY and ZP analyzed the data. JM and JL drafted the manuscript. $R L, G L$ and $Z P$ revised the manuscript. ZP coordinated the study. All authors have read and approved the final manuscript version

\section{Funding}

This work was supported by the Natural Science Foundation of Jiangsu Province, China (Grant No. BK20191309) and Shanghai Agriculture Applied Technology Development Program (grant number G2016060201) and the project funded by the Priority Academic Program Development of Jiangsu Higher Education Institutions (PAPD). The funders had no use in study design, data collection and analysis, decision to publish or preparation of the manuscript.

Availability of data and materials

All data generated or analysed during this study are included in this published article and its supplementary information files.

Ethics approval and consent to participate

All animal experiments in this study were carried out in the Laboratory Animal Center of Nanjing Agricultural University and approved by Laboratory Animal Monitoring Committee of Jiangsu Province, China [Permitted number: SYXK (SU) 2017-0007]. All efforts were made to minimize suffering. 


\section{Consent for publication}

Not applicable.

\section{Competing interests}

The authors declare that they have no competing interests.

\section{Author details}

${ }^{1}$ College of Veterinary Medicine, Nanjing Agricultural University, Nanjing 210095, China. ${ }^{2}$ OIE Reference Laboratory for Swine Streptococcosis, Nanjing 210095, China. ${ }^{3}$ South Dakota State University, Brookings, SD 57007, USA. ${ }^{4} \mathrm{MOE}$ Joint International Research Laboratory of Animal Health and Food Safety, Nanjing, China.

\section{Received: 11 April 2019 Accepted: 26 September 2019} Published online: 28 October 2019

\section{References}

1. Palmieri C, Varaldo PE, Facinelli B. Streptococcus suis, an emerging drugresistant animal and human pathogen. Front Microbiol. 2011;2:235.

2. Wertheim HF, Nghia HD, Taylor W, Schultsz C. Streptococcus suis: an emerging human pathogen. Clin Infect Dis. 2009;48(5):617-25.

3. Huang J, Shang K, Kashif J, Wang L. Genetic diversity of Streptococcus suis isolated from three pig farms of China obtained by acquiring antibiotic resistance genes. J Sci Food Agric. 2015;95(7):1454-60.

4. Huang J, Ma J, Shang K, Hu X, Liang Y, Li D, Wu Z, Dai L, Chen L, Wang L. Evolution and diversity of the antimicrobial resistance associated Mobilome in Streptococcus suis: a probable Mobile genetic elements reservoir for other streptococci. Front Cell Infect Microbiol. 2016;6:118.

5. Bernard R, El Ghachi M, Mengin-Lecreulx D, Chippaux M, Denizot F. BcrC from Bacillus subtilis acts as an undecaprenyl pyrophosphate phosphatase in bacitracin resistance. J Biol Chem. 2005;280(32):28852-7.

6. Eppelmann K, Doekel S, Marahiel MA. Engineered biosynthesis of the peptide antibiotic bacitracin in the surrogate host Bacillus subtilis. J Biol Chem. 2001;276(37):34824-31.

7. Wei S, Gutek A, Lilburn M, Yu Z. Abundance of pathogens in the gut and litter of broiler chickens as affected by bacitracin and litter management. Vet Microbiol. 2013;166(3-4):595-601.

8. Collinder E, Cardona ME, Berge GN, Norin E, Stern S, Midtvedt T. Influence of zinc bacitracin and Bacillus licheniformis on microbial intestinal functions in weaned piglets. Vet Res Commun. 2003;27(7):513-26.

9. Manson JM, Keis S, Smith JM, Cook GM. Acquired bacitracin resistance in enterococcus faecalis is mediated by an $A B C$ transporter and a novel regulatory protein, BcrR. Antimicrob Agents Chemother. 2004;48(10):3743-8.

10. Ohki R, Giyanto TK, Masuyama W, Moriya S, Kobayashi K, Ogasawara N. The BceRS two-component regulatory system induces expression of the bacitracin transporter, BceAB, in Bacillus subtilis. Mol Microbiol. 2003;49(4): $1135-44$.

11. Radeck J, Gebhard S, Orchard PS, Kirchner M, Bauer S, Mascher T, Fritz G. Anatomy of the bacitracin resistance network in Bacillus subtilis. Mol Microbiol. 2016;100(4):607-20.

12. Ouyang J, Tian XL, Versey J, Wishart A, Li YH. The BceABRS four-component system regulates the bacitracin-induced cell envelope stress response in Streptococcus mutans. Antimicrob Agents Chemother. 2010;54(9):3895-906.

13. Tsuda H, Yamashita Y, Shibata Y, Nakano Y, Koga T. Genes involved in bacitracin resistance in Streptococcus mutans. Antimicrob Agents Chemother. 2002;46(12):3756-64.

14. Charlebois A, Jalbert LA, Harel J, Masson L, Archambault M. Characterization of genes encoding for acquired bacitracin resistance in Clostridium perfringens. PLoS One. 2012;7(9):e44449.

15. Rodrigues JC, Caseli L. Incorporation of bacitracin in Langmuir films of phospholipids at the air-water interface. Thin Solid Films. 2017;622:95-103.

16. Zhu Y, Dong W, Ma J, Zhang Y, Pan Z, Yao H. Utilization of the ComRS system for the rapid markerless deletion of chromosomal genes in Streptococcus suis. Future Microbiol. 2019;14:207-22.

17. Pan Z, Liu J, Zhang Y, Chen S, Ma J, Dong W, Wu Z, Yao H. A novel integrative conjugative element mediates transfer of multi-drug resistance between Streptococcus suis strains of different serotypes. Vet Microbiol. 2019;229:110-6.

18. Pan Z, Ma J, Dong W, Song W, Wang K, Lu C, Yao H. Novel variant serotype of streptococcus suis isolated from piglets with meningitis. Appl Environ Microbiol. 2015;81(3):976-85.
19. Holden MT, Hauser H, Sanders M, Ngo TH, Cherevach I, Cronin A, Goodhead I, Mungall K, Quail MA, Price C, et al. Rapid evolution of virulence and drug resistance in the emerging zoonotic pathogen Streptococcus suis. PLoS One. 2009;4(7):e6072.

20. Chen C, Tang J, Dong W, Wang C, Feng Y, Wang J, Zheng F, Pan X, Liu D, Li $M$, et al. A glimpse of streptococcal toxic shock syndrome from comparative genomics of S. suis 2 Chinese isolates. PloS One. 2007;2(3):e315.

21. Ye CY, Zheng $H$, Zhang J, Jing HQ, Wang $L$, Xiong $Y W$, Wang $W$, Zhou ZM, Sun QZ, Luo X, et al. Clinical, experimental, and genomic differences between intermediately pathogenic, highly pathogenic, and epidemic Streptococcus suis. J Infect Dis. 2009;199(1):97-107.

22. Teng L, Dong X, Zhou Y, Li Z, Deng L, Chen H, Wang X, Li J. Draft Genome Sequence of Hypervirulent and Vaccine Candidate Streptococcus suis Strain SC19. Genome Announc. 2017;5(3):e01484-16.

23. Wang X, Zhao J, Cai C, Tang X, Fu L, Zhang A, Han L. A label-free quantitative proteomic analysis of mouse neutrophil extracellular trap formation induced by Streptococcus suis or Phorbol Myristate acetate (PMA). Front Immunol. 2018;9:2615.

24. Wu ZF, Wang WX, Tang M, Shao J, Dai C, Zhang W, Fan HJ, Yao HC, Zong J, Chen D, et al. Comparative genomic analysis shows that Streptococcus suis meningitis isolate SC070731 contains a unique $105 \mathrm{~K}$ genomic island. Gene. 2014;535(2):156-64.

25. Clifton-Hadley FA. Studies of Streptococcus suis type 2 infection in pigs. Vet Res Commun. 1984;8(3):217-27.

26. Vecht U, Wisselink HJ, Stockhofe-Zurwieden N, Smith HE. Characterization of virulence of the Streptococcus suis serotype 2 reference strain Henrichsen S 735 in newborn gnotobiotic pigs. Vet Microbiol. 1996;51(1-2):125-36.

27. Zhang A, Yang M, Hu P, Wu J, Chen B, Hua Y, Yu J, Chen H, Xiao J, Jin M. Comparative genomic analysis of Streptococcus suis reveals significant genomic diversity among different serotypes. BMC Genomics. 2011;12:523.

28. Wei WANG, Kong-wang HE, Yan-xiu NI, Li-xin L, Jun-ming ZHOU, ZHANG Xh, Bin LI. mRNA level of inflammation-associated cytokines of porcine alveolar macrophages cell lines 3D4/21 stimulated by Streptococcus suis serotype 2. Jiangsu J Agric Sci. 2013;3:017.

29. Ma YL, Lu CP. Isolation and identification of a bacteriophage capable of infecting Streptococcus suis type 2 strains. Vet Microbiol. 2008;132(3-4):340-7.

30. Vecht $U$, Arends JP. Differences in virulence between two strains of Streptococcus suis type II after experimentally induced infection of newborn germ-free pigs. Am J Vet Res. 1989;50(7):1037-43.

31. Hu P, Yang M, Zhang A, Wu J, Chen B, Hua Y, Yu J, Xiao J, Jin M. Complete genome sequence of Streptococcus suis serotype 14 strain JS14. J Bacteriol. 2011;193(9):2375-6.

32. Zhou Y, Dong X, Li Z, Zou G, Lin L, Wang X, Chen H, Gasser RB, Li J. Predominance of Streptococcus suis ST1 and ST7 in human cases in China, and detection of a novel sequence type, ST658. Virulence. 2017;8(6):1031-5.

33. Athey TB, Auger JP, Teatero S, Dumesnil A, Takamatsu D, Wasserscheid J, Dewar K, Gottschalk M, Fittipaldi N. Complex population structure and virulence differences among serotype 2 Streptococcus suis strains belonging to sequence type 28. PLoS One. 2015;10(9):e0137760.

34. Tang JQ. Analyses reveal a novel avirulent Streptococcus suis serotype 2 strain that induces protective immunity against challenge with the highly virulent strains. Retrovirology. 2010;7:66.

35. Athey TB, Vaillancourt K, Frenette M, Fittipaldi N, Gottschalk M, Grenier D. Distribution of Suicin gene clusters in Streptococcus suis serotype 2 belonging to sequence types 25 and 28. Biomed Res Int. 2016;2016:6815894.

36. Wang K, Chen J, Yao H, Lu C. Whole-Genome Sequence of Streptococcus suis Serotype 3 Strain YB51. Genome Announc. 2013;1(6):e00884-13.

37. Wang K, Chen J, Yao H, Lu C. Whole-Genome Sequence of Streptococcus suis Serotype 4 Reference Strain 6407. Genome Announc. 2014;2(4):e00770-14.

38. Yu WL, Li CL, Wang GP, Lu CP. Pathogenic characteristics of Streptococcus suis type 2 and type 9 isolated from Guangdong province. Vet Sci China. 2007;37(8):650-4.

39. Wang K, Yao H, Lu C, Chen J. Complete Genome Sequence of Streptococcus suis Serotype 16 Strain TL13. Genome Announc. 2013;1(3):e00394-13.

40. Lai L, Dai J, Tang H, Zhang S, Wu C, Qiu W, Lu C, Yao H, Fan H, Wu Z. Streptococcus suis serotype 9 strain GZ0565 contains a type VII secretion system putative substrate EsxA that contributes to bacterial virulence and a vanZ-like gene that confers resistance to teicoplanin and dalbavancin in Streptococcus agalactiae. Vet Microbiol. 2017;205:26-33.

41. Jobin MC, Grenier D. Identification and characterization of four proteases produced by Streptococcus suis. FEMS Microbiol Lett. 2003;220(1):113-9. 
42. Zheng H, Du P, Qiu X, Kerdsin A, Roy D, Bai X, Xu J, Vela Al, Gottschalk M. Genomic comparisons of Streptococcus suis serotype 9 strains recovered from diseased pigs in Spain and Canada. Vet Res. 2018;49(1):1.

43. Hu P, Yang M, Zhang A, Wu J, Chen B, Hua Y, Yu J, Chen H, Xiao J, Jin M. Complete genome sequence of Streptococcus suis serotype 3 strain ST3. J Bacteriol. 2011;193(13):3428-9.

44. Zhang Y, Lu P, Pan Z, Zhu Y, Ma J, Zhong X, Dong W, Lu C, Yao H. SssP1, a Streptococcus suis Fimbria-Like Protein Transported by the SecY2/A2 System, Contributes to Bacterial Virulence. Appl Environ Microbiol. 2018; 84(18):e01385-18.

45. Pan Z, Ma Y, Ma J, Dong W, Yao H. Acute meningitis of piglets and mice caused by co-infected with Streptococcus suis and Aerococcus viridans. Microb Pathog. 2017;106:60-4.

46. Wu Z, Wu C, Shao J, Zhu Z, Wang W, Zhang W, Tang M, Pei N, Fan H, Li J, et al. The Streptococcus suis transcriptional landscape reveals adaptation mechanisms in pig blood and cerebrospinal fluid. Rna. 2014;20(6):882-98.

47. Livak KJ, Schmittgen TD. Analysis of relative gene expression data using real-time quantitative PCR and the 2(-Delta Delta C(T)) method. Methods. 2001;25(4):402-8.

\section{Publisher's Note}

Springer Nature remains neutral with regard to jurisdictional claims in published maps and institutional affiliations.

Ready to submit your research? Choose BMC and benefit from:

- fast, convenient online submission

- thorough peer review by experienced researchers in your field

- rapid publication on acceptance

- support for research data, including large and complex data types

- gold Open Access which fosters wider collaboration and increased citations

- maximum visibility for your research: over $100 \mathrm{M}$ website views per year

At $\mathrm{BMC}$, research is always in progress.

Learn more biomedcentral.com/submissions 